STUDI

FRANCESI

\section{Studi Francesi}

Rivista quadrimestrale fondata da Franco Simone

190 (LXIV | I) | 2020

Varia - fasc. I - gennaio-aprile 2020

\title{
Manuscript to Print, Print to Digital
}

\section{Maria Colombo Timelli}

\section{OpenEdition}

Journals

Édition électronique

URL : https://journals.openedition.org/studifrancesi/22307

DOI : $10.4000 /$ studifrancesi.22307

ISSN : 2427-5856

Éditeur

Rosenberg \& Sellier

\section{Édition imprimée}

Date de publication : 1 avril 2020

Pagination : 150-151

ISSN : 0039-2944

\section{Référence électronique}

Maria Colombo Timelli, « Manuscript to Print, Print to Digital », Studi Francesi [En ligne], 190 (LXIV | I) |

2020, mis en ligne le 01 avril 2020, consulté le 03 août 2021. URL : http://journals.openedition.org/ studifrancesi/22307 ; DOI : https://doi.org/10.4000/studifrancesi.22307

\section{Ce document a été généré automatiquement le 3 août 2021.}

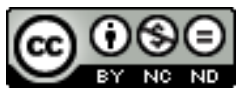

Studi Francesi è distribuita con Licenza Creative Commons Attribuzione - Non commerciale - Non opere derivate 4.0 Internazionale. 


\title{
Manuscript to Print, Print to Digital
}

\author{
Maria Colombo Timelli
}

\section{RÉFÉRENCE}

Manuscript to Print, Print to Digital, “Le Moyen Français" 81, 2017 (Part 1); 82, 2018 (Part

2)

1 Un colloque qui s'est tenu en 2016 au Château de la Bretesche est à l'origine de deux fascicules du «Moyen Français»; les cinq contributions publiées dans la première issue portent sur le paratexte des éditions anciennes $\left(\mathrm{XV}^{\mathrm{e}}\right.$ et $\mathrm{xvI}^{\mathrm{e}}$ siècles) interprété comme lieu d'une «performance éditoriale» (Armstrong, Brown, Dauvois), sur les pratiques d'édition actuelles appliquées à des «performances textuelles», pièces théâtrales ou musicales entre autres (van Orden, Winn), alors que le deuxième fascicule s'organise en deux parties, l'une consacrée à l'édition des pièces théâtrales (Koopmans, Duhl), l'autre aux pratiques éditoriales appuyées aux nouvelles technologies (Cornilliat, Legaré \& Memmi).

2 Adrian ARMSTRong, L2 Editorial Identity Performance. French-Language Publication in DutchSpeaking Environments in the Burgundian Low-Countries, 81, pp.11-40. Les éditeurs néerlandais des anciens Pays-Bas bourguignons ne se sont pas privés de publier de nombreux ouvrages en français: A.A. revient sur un phénomène bien connu pour souligner comment ces éditions anciennes (son corpus couvre les années 1480-1530) associent des pratiques parfois contradictoires: des caractères typographiques (la «textura») qui renvoient à la production en néerlandais, la langue française des textes publiés, des colophons et des illustrations rapportés eux aussi au monde néerlandophone. Deux œuvres de Jean Lemaire de Belges publiées à Anvers en 1508 et en 1509 par deux éditeurs différents confirment ces traits tout à fait particulier de la production éditoriale de cette région.

Cynthia J. BRown, The Drama of the Visual Editing of Pierre Gringore's "Chasteau de Labour", 81, pp. 41-74. La première œuvre de Pierre Gringore a joui d'une fortune certaine entre 1501 et la fin $\mathrm{du} \mathrm{XVI}^{\mathrm{e}}$ siècle, témoignée par une vingtaine d'éditions connues (liste en 
annexe, pp. 72-74). C.J.B. étudie le rapport texte-image dans ce poème allégorique, en insistant, d'une part, sur le caractère théâtral de la pièce, d'autre part sur l'interprétation «dramatique» suggérée par les programmes iconographiques euxmêmes. Une attention particulière est consacrée à l'édition de Philippe Pigouchet et Simon Vostre (1501, la dernière sous le contrôle de l'auteur), illustrée tant par des gravures sur bois que par des gravures sur cuivre, puis aux éditions de Gaspard Philippe et Nicolas de la Barre (1502-1505), qui reproduisent le programme de Pigouchet Vostre, aux impressions sorties de l'atelier Trepperel, dans lesquelles le rapport avec le texte est certainement moins soigné, et enfin aux éditions d'Alain Lotrian (ca 1520) et Claude Nourry (Lyon, 1526), qui, tout en reproduisant au moins en partie le programme de Pigouchet - Vostre, s'en éloignent en introduisant des rubriques plus développées. La dernière édition considérée, pour Galliot du Pré (1532), adopte une présentation résolument «moderne» (caractères romains, ponctuation, accents, numérotation des pages, titres courants) d'où les images disparaissent.

4 L'article de Nathalie DAuvors (Voices on the Page: Brant, Locher, Badius, 81, pp. 75-105) concerne la mise en page de quelques éditions de Térence, Horace, Ovide, par Sebastian Brant, Jacob Locher et Josse Bade, publiées entre 1493 et 1503 (liste aux pp. 103-105).

Kate VAN ORDEN, The Voice in the The Text. Recovering Sixteenth-Century Vernaculars, 81, pp.109-132. L'édition des pièces musicales soulève des questions spécifiques aux musicologues. K.v.O. s'appuie sur deux textes en particulier: la chanson de Clément Janequin L'Alouette (editio princeps, Paris, ca 1528), dont les vers doivent être «corrigés» si tant est que l'éditeur veut obtenir un poème rythmiquement acceptable à l'écrit, et la chanson Vray Dieu quel paine esse de Loyset Compère (début XVI ${ }^{e}$ siècle), dont la plupart des manuscrits sont d'origine italienne; dans ce dernier cas, au-delà des graphies italianisantes, les variantes linguistiques transmises par ces copies s'avèrent tout à fait compatibles avec les régionalismes attestés par les linguistes de l'époque, notamment par Meigret.

6 Mary Beth winN (Verse Lais in the Prose "Tristan", pp. 81, 133-175) s'intéresse à la mise en page de trois lais dans le Tristan en prose: les deux Lais mortels composés l'un par Tristan, l'autre par Iseut, et le Lai voir disant / mesdisant de Dinadan qui conclut le roman. En s'intéressant en particulier aux éditions imprimées, dues à Antoine Vérard 1489, ca 1496, ca 1499, ca 1506, puis à Michel Le Noir 1514 et 1520, et enfin à Denis Janot 1533, elle montre comment la présentation infléchit la perception visuelle des poèmes auprès des lecteurs: chez Vérard les vers sont imprimés sur longues lignes, alors que Michel Le Noir revient à la mise en page de la plupart des manuscrits, avec des alinéas pour chaque vers. Si l'absence de notation musicale ne surprend pas (on ne la retrouve que dans deux manuscrits de la fin du xiII e siècle), la composition de lais lyriques demeure un trait distinctif tant de l'auteur anonyme que de ses protagonistes. En annexe: liste des imprimés avec lieux de conservation, description synthétique des manuscrits cités, édition des trois lais objet de l'article.

7 La contribution de Jelle KoOPMANs (Farces and "Sotties". Performance Practice, Print Practice, and Manuscript Practice, 82, pp. 3-20) se veut une réflexion de méthode sur le traitement et l'usage à faire des documents parvenus jusqu'à nous et sur lesquels les historiens se basent pour reconstruire une histoire du théâtre français. Les questions principales ne portent pas uniquement sur le contenu des documents - y compris les textes des pièces consignés dans des manuscrits ou des imprimés -, mais aussi, et surtout, sur les raisons pour lesquelles ces documents ont été conservés. La quantité très limitée de ces 
documents mêmes, qui risquent de fausser notre vision de l'histoire et du théâtre médiéval, est un aspect primordial, que la critique a tout intérêt à ne jamais oublier.

C'est à une pièce en particulier qu'est consacré l'article d'olga Anna DUHL (Satire and History in Performance. A New Edition of the "Sotye nouvelle des croniqueurs" (Pierre Gringore ?, c. 1515), 82, pp. 21-53). Il s'agit de discuter d'abord de l'attribution au rhétoriqueur et dramaturge Pierre Gringore, de présenter les éditions modernes de la Sotye et surtout d'en discuter l'interprétation sur la base du contexte historique au moment de l'avènement de François $\mathrm{I}^{\mathrm{er}}$. En soulignant en particulier les similarités des premiers vers des Fantasies de Mere Sote du même Gringore avec une cinquantaine de vers de la Sotye, O.A.D. prouve, sinon l'attribution des deux œuvres au même auteur, au moins la circulation des textes et leur stratification dans le milieu théâtral à cheval entre $\mathrm{xv}^{\mathrm{e}}$ et $\mathrm{XVI}^{\mathrm{e}}$ siècle.

9 En préparant son édition du Panegyric du Chevallier sans reproche (1527, biographie poétique de Louis de la Tremoïlle, mort à Pavie), François CoRNILLIAT a pu s'appuyer sur internet afin de repérer une grande partie des sources exploitées par Jean Bouchet; la liste en est longue, et comprend, outre les auteurs cités par le poète lui-même (Valère Maxime, Boccace, Sabellico): Alain Chartier, Claude de Seyssel, Ravisius Textor, Francesco Patrizi, Battista Fregoso, Bartolomeo Sacchi / il Platina. Si les nouvelles méthodes peuvent rendre de grands services aux éditeurs critiques, surtout en abrégeant leurs recherches, la littérature - et notre connaissance des textes des siècles passés - demeure, quant à elle, un objet qu'aucune approche méchanique ne permettrait d'atteindre en tant que tel (Cut-and-Paste, Then and Now, On Editing Jean Bouchet's "Panegyric du Chevallier sans reproche", and some Related Matters, 82, pp. 57-74).

10 Une dernière étape vers les nouveaux media est franchie par Anne-Marie LEGARÉ et Paul MEMMI, qui ont créé un «JEAM» («Jeu des Échecs amoureux moralisés») à partir du Livre des Échecs amoureux moralisés d'Évrard de Conty (inter 1370 et 1400). Le but en est d'offrir une approche renouvelée à une œuvre de la fin du Moyen Âge que son auteur lui-même avait conçue comme «multimedia», basée sur les ressources de son temps: mise en page, texte, enluminures, sans oublier le sujet qui en constitue le cœur, à savoir une partie d'échecs pouvant bien être réalisée (From Manuscript to Digital. Conceptualizing a Ewbdoc Version of "Le Livre des échecs amoureux moralisés", 82, pp. 75-96).

11 Une bibliographie d'ensemble, commune aux deux fascicules, est organisée en trois sections: sources primaires, sources secondaires et enregistrements musicaux (pp. 97-123); elle est suivie de l'index des manuscrits (pp. 125-127). 